\title{
Hysterical paraplegia
}

\author{
J H E BAKER,* J R SILVER $\dagger$
}

From the Rookwood Hospital,* Cardiff, Wales, and National Spinal Injuries Centre, $\uparrow$ Stoke Mandeville Hospital, Aylesbury, Bucks, UK

SUMMARY Between 1944 and 198420 patients were admitted to a spinal injuries centre with a diagnosis of traumatic paraplegia. They subsequently walked out and the diagnosis was revised to hysterical paraplegia. A further 23 patients with incomplete traumatic injuries, who also walked from the centre, have been compared with them as controls. The features that enabled a diagnosis of hysterical paraplegia to be arrived at were: (1) They were predominantly paraplegic, (2) There was a high incidence of previous psychiatric illness and employment in the Health Service or allied professions, (3) Many were actively seeking compensation. The physical findings were a disproportionate motor paralysis, non anatomical sensory loss, the presence of downgoing plantar responses, normal tone and reflexes. They made a rapid total recovery. In contrast, the control traumatic cases showed an incomplete recovery and a persistent residual neurological deficit. Investigations apart from plain radiographs of the spinal column were not warranted, and the diagnosis should be possible on clinical grounds alone.

The diagnosis of non organic paraplegia is not easy. Problems arise with this diagnosis because of difficulties with psychiatric terminology and uncertainty as to outcome. Cases considered on psychiatric grounds to be classical cases of hysterical paraplegia have subsequently turned out to have organic disease. Thus the outcome of a complete recovery must be used as one of the criteria for establishing the diagnosis confidently as being non organic. There is a separate and more common phenomenon of elaboration of real deficit which we will not analyse in this paper.

The practical question is "how does the physician diagnose the patient likely to have a feigned or non organic paraplegia?" Between 1944 and 1981, 20 patients with non organic paralysis were referred to the National Spinal Injuries Centre (NSIC) from referring hospitals having been completely investigated and considered to have traumatic spinal cord injuries. These patients subsequently recovered completely and walked out of the National Spinal Injuries Centre, after they had been diagnosed as having hysterical paraplegia. We have therefore studied these 20 patients and in addition three others who were consecutively referred to Spinal Injuries Centres associated with one of the authors, JHB. This total

Address for reprint requests: $\mathrm{Dr} \mathbf{J}$ R Silver, Stoke Mandeville Hospital, Aylesbury, Bucks HP21 8AL, UK.

Received 14 February 1984 and in revised form 24 July 1986. Accepted 26 July 1986 group is termed the "non organic" group. We have compared the clinical findings, investigations and natural history with a further 23 control patients also seen by $\mathrm{JHB}$, in whom there was no doubt that there was a traumatic spinal cord injury. These also made a recovery to the extent of being able to walk.

We have attempted to isolate the features of history, examination and investigation which differ between the two groups which might be useful in predicting non organic paraplegia. Whether these features conform to the accepted criteria for the diagnosis of Hysteria is open to discussion. Indeed, in practice, whether these patients have an isolated conversion reaction, are malingerers, or are merely immature personalities reacting to a life situation with a feigned neurological complaint is often impossible to deduce.

\section{Material and incidence}

Twenty patients were admitted or seen as outpatients at the NSIC. They were initially thought to be cases of traumatic paraplegia but subsequently the diagnosis of non organic paraplegia was made at Stoke Mandeville between 1944 and 1981. A further three patients were similarly seen at Oswestry in 1984.

The 20 Stoke Mandeville cases were seen among a total of 7000 cases of which 5000 were traumatic and 2000 diagnosed as medical cases of paraplegia, for example multiple sclerosis or tumour. The three cases at Oswestry were seen over a two year period; the referral rate being 80 traumatic and 20 medical cases per annum. 
The control patients were provided by 23 patients who walked out of a centre having recovered from a traumatic spinal cord injury. In these patients we were confident that an organic lesion had been sustained by virtue of a vertebral fracture or an appropriate injury associated with bruising, tenderness, soft tissue injury and associated injuries combined with the clinical picture.

\section{Sex distribution}

The distribution of the non organic group was 14:9 (male:female). The distribution for traumatic paraplegia was 20:3 (male:female). We have been unable to match the sex distribution in our control patients.

\section{Injury}

All patients in both series identified an injury to which they related the start of their symptoms. No difference in declared severity was noted. Some of the patients in the non organic group had falls of a minor nature which could support a diagnosis of an extension injury but this was only in retrospect following radiography. It was difficult to reconcile the injury with the severity of the paralysis. In contrast in the organic group, the controls, it was always possible to reconcile the severity with the mechanism of the injury and with the clinical picture.

\section{Associated injury}

There was no associated injury in the non organic group. In control cases, there was the appropriate associated trauma of a head injury associated with a cervical injury, fractured ribs seen in thoracic injuries and fractured long bones. One patient had an associated brachial plexus lesion and another a bulbar palsy. The brachial plexus injury was seen in a motor cyclist and arose from impacting on his shoulder when decanted from his machine. The bulbar lesion related to a very incomplete high cervical lesion and remitted completely.

\section{Time of presentation}

All non organic cases were referred to us in the immediate period (up to four months) following injury. One patient was admitted on two occasions with a paraplegia following separate accidents. The first time he was admitted within days and the second time after an interval of 4 months following a shipboard incident in Dubai. All our control series were referred in the immediate period also. We also had available the case records of the primary referring hospitals and could review the signs presenting in casualty and on their wards as well as when we first saw them.

\section{Level of paralysis}

All non organic patients presented initially with a paraplegia and there were no primary tetraplegics. Two presented secondarily; one woman was admitted from the Stoke Mandeville Games, having sustained a trivial injury when someone banged the back of her wheelchair. Her upper limb symptoms remitted within 36 hours and she returned to competition; paraplegic as before. When we informed her "parent" spinal centre, they replied that they considered her paraplegia to be functional also. The second case was a merchant seaman who had presented with a paraplegia after his first accident, made recovery and went back to sea. There, following a shipboard fall, he became tetraplegic. This was also non organic and once more he made complete recovery.

In the control group, there were nine cervical injuries and 14 dorsal and lumbar injuries.

\section{Employment: male}

Four non organic patients were serving in the Armed Forces. One was a merchant seaman and one was a policeman. Two patients were prisoners. One was working in the Health Service as a porter. The distribution in the control group showed no pattern other than that dictated by age ( $25 \%$ were schoolboys/students). There were no armed or public service employees. None were in legal custody and only two were unemployed.

\section{Employment: female}

Four of the nine females were involved in the health system. All were Registered Nurses. One was a servicewoman. No health workers were in the control female group; this was too small to draw any direct conclusions.

The high incidence of five males and females employed within the health service out of a sample of 23 contrasts with the absence of any in the control group.

\section{PAST MEDICAL HISTORY}

Medical In the non organic group, one patient suffered from Addison's disease and had had tuberculosis in the past. The control group was little different; there was one case of tuberculosis and an asymptomatic ventriculoseptal defect. Orthopaedic One non organic patient without underlyingo bone disease had sustained nine separate accidents producing broken bones. Another male had had four operations on the same knee. Two control patients had had one fracture each, both occurred in association with previous motor cycle accidents.

Gynaecological In the non organic group one woman had had a previous hysterectomy: another was demanding a hysterectomy from her medical attendants; she had been raped at the age of eight and had given birth to several malformed infants. There had been two other gynaecological procedures. None had been sterilised. No gynaecological operations took place on any of the women in the control group. As the numbers were not sex matched, no significance can be drawn from these figures.

\section{PSYCHIATRICILLNESS}

Seven non organic patients had previously been admitted to mental hospitals. The details are in table 1. The control group had two patients with psychiatric disturbance. One patient had had ECT 25 years earlier and had been well since. The other was suicidal from a depressive psychosis and her spinal cord injury was due to self precipitation from a window in a suicide attempt.

\section{Table 1 Psychiatric hospital admission}

Admitted for intruding foreign bodies Prisoner

LSD abuse

Depressive illness

Admission for narcosis therapy

Persecution delusions 
Table 2 Previous functional disorder

\section{Non neurological}

Cardiac neurosis

Functional dyspnoea

Hyperventilation syndrome

Neurological

Foot drop

Paralysis

Field defect

Seizures

Bizarre ankle posturing

Dysphonia

Dysphagia

Dysgeusia

Anosmia

Table 3 Modelling factors

Previous spinal injury with full recovery

Previous stab wound of back: no neurological signs

Previous traction for muscular back injury

Paraplegic mother in law

Cohabiting catamite with paraplegia from DS

PREVIOUS FUNCTIONAL DISTURBANCE

Presentations with functional disorder had occurred before in 14 non organic patients and in 10 of these the symptoms had related to the central nervous system. The details are shown in table 2 . There were no previous functional disturbances in the control group.

\section{SPINAL-FAMILY HISTORY}

Five non organic patients had had previous injuries to the spine (non paralytic) resulting in full recovery or there had been spinal cord injury or non traumatic paralysis in their families or associates. The details appear in table 3 . One woman had "sprained her neck" in a previous hunting accident. There had been no bony, ligamentous or neurological damage and she had regarded this as "trivial". It was recalled only because it had been recent. No control patients had a past history of spinal trauma.

\section{SECONDARY GAIN}

In the non organic males, three servicemen were in the process of attempting to secure their discharge from the armed forces; two were prisoners; one was a victim of criminal violence; one was a policeman injured on duty; one man was avoiding arrest; one man was suing his employer for negligence; one was suing the driver of the car in which he was injured. In the remainder, no obvious cause of secondary gain was evident. Altogether 10 out of 14 cases had some potential for secondary gain.

In the women, three had reached an active stage in their matrimonial litigation; one was attempting to coerce the Service authorities to return her husband from the South Atlantic (a previous functional disorder had succeeded in securing his return from an unaccompanied overseas posting); one girl had been forced by her parents into a musical career for which she had no aptitude. Thus potential for gain was present in five out of nine cases.

In the control group, only four civil actions were pending; two were car passengers suing the driver, one for the Criminal Injuries Compensation Board and one Industrial Injury. In no case was liability in doubt. There were no pre existing criminal proceedings. Minor motoring proceedings
Table 4 Motor and reflex features

Jerky and erratic movements on formal testing

Agonist/antagonist contractions clinically

Inconsistency of groups in motor assessment

Bizarre functional posturing

Incompatible formal testing with observed acts

Normal motor tone and reflexes

Normal plantar responses

arose from two cases, both of which were eligible for disposal in absentia by mail. None of our control cases were involved in matrimonial proceedings, nor were any precipitated; nor did matrimonial disharmony present a problem in management.

The overall incidence of potential gain in the non organic group was 15 out of 23 ; in the organic group it was six out of 23.

SELF DISCHARGE

Five non organic patients took their own discharge and two others effectively did so by engineering events. The important feature was that none of them took their own discharge while still overtly paralysed: all made recovery before discharge and walked out of the unit. This was never seen in organic paraplegia and no patients in the control group did this.

\section{MULTIPLE FACTORS}

Of the last five features psychiatric illness, previous functional disturbance, spinal/family history, and secondary gain, all the non organic patients had at least two of these in their histories. In the control group, only seven had any features at all and none more than one.

\section{PHYSICAL EXAMINATION: MOTOR FEATURES}

All non organic patients had a complete motor paralysis when first seen after the provoking episode. However, three cases improved during transfer to us and had no motor signs on arrival. The features which we found helpful in identifying the cases of non organic paraplegia are given in table 4 . None of these were present in our control group.

Apart from those motor features seen during formal examination, in five cases there was marked incompatibility between the examination and observed or self declared activity. One patient was observed skipping in the physiotherapy department. One, with an ostensible triparesis, assisted the doctor taking blood with her allegedly paralysed arm. Another got herself back normally into a wheelchair having fallen out of it. Another, who had had to be supported between two nurses to move across the consulting room was observed walking without assistance to her car. Another having virtually to be carried across the consulting room was living satisfactorily, by her own admission, in a primitive cottage with an outside privy.

In the control group, four patients appeared to have complete loss of motor power and sensory appreciation in casualty, but some evidence of preservation of sensory function without the return of motor function had appeared by the time they arrived in the wards of the admitting hospital. No incompatibility between examination and function was seen in the organic control series.

\section{Reflex activity}

In the non organic group, the deep tendon reflexes were normal in 22 cases. One held the limbs rigid with 
Table 5 Clustering of motor features

\begin{tabular}{ll}
\hline No motor symptoms & 3 \\
No marker features & Nil \\
One marker feature & Nil \\
Two marker features & 7 \\
Three marker features & 5 \\
Four marker features & 3 \\
Five marker features & 5 \\
\hline
\end{tabular}

(marker features are those listed in table 4)

agonist/antagonist contractions and reflexes were not elicitable. There were no patients with enhanced or pathological reflexes. The plantar responses were normal (downgoing) in all cases. In the control group the most important diagnostic feature was the presence of an extensor plantar response. This was seen in 13 cases. In five it was absent, and in two it was downgoing. The tendon reflex was frequently absent at the level of the injury. Below the level they showed an orderly progression through the stage of spinal shock to become pathologically increased.

\section{Clustering}

All the non organic patients who were still paralysed at the time they were seen at the centre had two or more of the marker features. See table 5 .

PHYSICAL EXAMINATION: SENSORY FEATURES

Non organic The useful features seen on sensory examination were inconsistency and non reproducibility of sensory signs. Non-dermatomal circumferential truncal sensory loss was seen in 14 cases; three other cases had nonanatomical sensory disturbances; and in two cases there were consistently wrong responses on testing of joint position sense. Several of the features occurred grouped together. In three cases, sensation was reported as normal. In two cases none of these markers were found; in six cases one was found; in nine cases, two, and in three cases, three.

In the control group, all patients had some preservation of sensory function by the time they were examined in the ward of the receiving hospital. In 16 our of 23 cases, some posterior column function was seen; it was intact in eight. Sacral sparing was seen in five cases. All examination findings were consistent and in an anatomical distribution and, in the case of the central cord lesions, were compatible and appropriate to the level of the bony injury as shown by bruising, tenderness and the radiological findings.

\section{Wasting and pressure sores}

Wasting not seen in either group. No patient in our non organic series developed a pressure sore or pressure mark. The control group had three patients who developed sores by the time of admission to a spinal centre.

\section{AUTONOMICFEATURES}

Bladder disturbance No non organic patients had a primary disturbance of bladder function and all continued to pass urine normally after the precipitating incident. Four, who were not catheterised, despite complaints that they were in retention, voided normally in the Accident Department of the receiving hospital before transfer. Three patients arrived with catheters already inserted; these were removed on arrival and they were able to void and to initiate and suppress flow on command. Three patients developed secondary urological complaints having been placed in the spinal centre where routine bladder training was being carried out.
These cases show inconsistencies with other aspects of autonomic function such as wearing a condom and leg bag while able to initiate and suppress flow to command and with normal urodynamic studies. One woman, with recurrent episodic retention, was further investigated with myelography and this was negative. This secondary disturbance we regard as an example of acquired behaviour.

Incontinence was seen subsequently in two patients. One had ability to initiate and suppress flow to command and the other was a prisoner who had been ignored on a previous complaint of retention and had ultimately leapt out of bed and rushed to the lavatory to void, following which he had fallen to the ground at the base of the urinal, reputedly unable to move. Left there, he had surreptitiously crawled back to bed. The next time his bladder filled, he was incontinent in the bed. We regard both these as volitional.

All patients in the control group except two initially showed retention of urine and required catheterisation. In the two with preserved voiding, there was complete sacral sparing. In two other cases, who needed catheterisation, there was some bladder sensation without motor function and in these cases, there was some sacral sensation but short of normal. A final case which did have apparent normal sacral sensation was in retention but the conus reflexes were disturbed and urodynamic studies were abnormal.

Bowel disturbance In the non organic group, this was extremely rare and again showed inconsistencies. One youth performed manual evacuations despite all other sacral functions being normal; the other, despite being allegedly totally constipated, had no faecal masses on plain radiographs and at barium enema was able to expel the medium to command In the control group, the two patients with intact bladde? function also had intact bowel function. One patient with sacral sparing could feel rectal distention. All other patient required manual evacuations and laxatives with or without suppositories in the first instance.

Sexual function This was only rarely reported. One patient reported impotence in the presence of preserved normal bladder and bowel function. The other recovered his potency pari passu with sensorimotor function following a placebo injection of sterile water. The control group showed appropriate loss of erection and ejaculation consistent with their level of injury.

INVESTIGATIONS: PLAIN RADIOGRAPHS

Plain films of the spine were obtained in all cases. Only three of the non organic group were abnormal. One had the changes of osteomyelitis; one had a spina bifida and the third had evidence of a previous gunshot wound. The first case was investigated with myelography (negative). There was no evidence of acute injury to either bony or soft tissue. The second was not investigated further (there was recovery within 24 hours and the patient was a drug abuser). The last was incidental.

This was markedly different from the control group. A bony injury compatible with the last normal segment was found in all save seven cases. In six of these seven, there was evidence of a hyperextension injury and these were accompanied by the picture of a central cord lesion. In the last (a child) no definite injury could be seen on first films but on subsequent films, bony bridging at the appropriate level was evident. 


\section{Hysterical paraplegia}

INVESTIGATIONS: STUDIES OF CEREBROSPINAL FLUID Undertaken in six non organic cases, all were normal. All were examined before immune phoretic methods were available. This was not performed in the control cases.

INVESTIGATIONS: ELECTROPHYSIOLOGICAL

Evoked responses were undertaken in three non organic patients in connection with medicolegal reports. All were normal. One of the patients, who knew we felt his lesion was functional, defaulted from his first appointment, the night before being admitted to his local hospital with "seizures" which the attending neurologist felt were functional also. Electromyography also showed agonist/antagonist contractions. These were not performed in the control group. Sphincter EMGs were however performed in selected urodynamic studies. All were abnormal.

\section{INVESTIGATIONS: MYELOGRAPHY}

Myelography was undertaken in six non organic cases, four before arrival in the spinal centre and two after. All were normal. We undertook myelography in one patient because of significant abnormality on plain films, (osteomyelitis); and in the other because of episodic recurrent retention at OP follow up. Myelography was undertaken in other hospitals in five control cases and CT scanning in one prior to surgery. All showed either a block to contrast flow or canal narrowing by bone fragments.

\section{SURGERY}

This was not undertaken as either a therapeutic or diagnostic tool. Two patients had presented with paralysis, however, following negative laminectomies for neurological symptoms without, according to the submitted record, any neurological signs. In the control series, three patients were finally operated on: two spinal fusions and one decompression. One patient deteriorated after surgery transiently.

\section{FAR A DISM}

Treatment

This produced only one cure and this was thought to be due to the pain produced. Used in two other cases, it produced bizarre movements and posturings but had no therapeutic effect.

\section{Suggestion, abreaction and placebo}

Suggestion was used specifically in five cases, producing good results in all. Intravenous pentothal produced improvement in one case and made no difference in the other. A placebo injection of sterile water was used successfully in one man, a teenage soldier from rural Libya. Manipulation was used successfully in one case, and one man responded to the arbitrary injunction to "Get up". In the majority of patients, no specific treatment was used. The patient was given a diagnosis of "cord concussion" and this was coupled with a firm confident prediction that recovery would shortly take place.

In the control group, this treatment clearly was not matched or controlled. They were admitted to the spinal centre and received the appropriate treatment for their fractures.
Table 6 Time for complete recovery in non organic cases

\begin{tabular}{ll}
\hline On arrival/within one day & 4 \\
1 day & 3 \\
2 days & 8 \\
5 days & 2 \\
6 days & 1 \\
7 days & 1 \\
16 days & 2 \\
17 days & 1 \\
\hline
\end{tabular}

\section{NATURAL HISTORY}

The pattern of recovery in the non organic cases is shown in table 6 . All had recovered completely within 18 days; thirteen within the first $\mathbf{4 8}$ hours and all apart from four within a week. Improvement in the sensorimotor function occurred together and often in either global or random pattern.

In the control group, four patients deteriorated compared with the findings at the accident site following the first neurological examination. Two patients walked about at the roadside after injury and one deteriorated after being stood 24 hours after injury for fitting of a doll's collar. These cases had started to improve within hours of the precipitant event and finished up better than the original level. The patients improved in slower time than the non organic cohort, and sensory improvement occurred first, with motor second and autonomic last. Proximal improvement preceded distal, and lower limb in cervical cases, preceded upper (probably due to the high incidence of central cord injuries).

FOLLOW UP: NON ORGANIC CASES

It is not our policy to give formal follow up appointments within the spinal centre to patients in whom a diagnosis of non organic paraplegia has been made because of the risks of developing acquired behaviour. The general practitioners and referring hospitals were contacted by post and followed up any untoward events. Sixteen of our cases have either been seen or reports have been received. Two patients developed functional seizures. One developed epilepsy, confirmed by electroencephalography, no cause being found. One patient, after return to her mental institution, relapsed. She was seen by a consultant neurosurgeon who reported a sensory level "going up and down like a yoyo". Two patients developed secondary episodic retention of urine; their management is reported in the section on autonomic disturbance, but no organic lesion was found. One patient, living close to Stoke Mandeville, has become a cardiac neurotic and has had multiple admissions to our Coronary Unit with chest pain but no evidence of either cardiac ischaemias or myocardial infarction.

Syndrome shift has occurred in three others, developing either chest pain in one or asthma in two. Three have been seen for performance of spinal evoked responses for medicolegal purposes. One reported to the physiotherapy department demanding a wheelchair and a disabled parking sticker while still walking normally and with no signs. One patient telephoned to announce she had a pressure sore; a visit by the general practitioner revealed her to be telling falsehoods. The last was seen by one of us (JHB) out canoeing with junior servicemen some 9 months later; she had, she said, celebrated her discharge from the Spinal Unit by going rafting with the service apprentices the same day down the 
River Severn! No new autonomic disturbances have been reported in this group.

FOLLOW UP: CONTROL CASES

The control group were all followed up for a period between one and 3 years. All patients walked without any aids. Power deficits could be elicited on formal testing in some and five patients had increased tone and spasticity. All patients had permanently abnormal deep tendon or superficial reflexes and/or extensor plantar responses. Residual sensory disturbance in one modality (blunting to pin prick) was seen in four. Intractible pain was a feature in three cases. Persistent disturbance of bladder bowel and sensory function were seen in the majority of cases. Of the 15 adult males, five stated that their sexual function (sensation, erection and ejaculation) is entirely normal. None has admitted to siring children as yet. Despite their disability, only three patients have not returned to previous occupations.

\section{Discussion}

Our numbers would confirm that non organic paraplegia is rare. When comparison is made with the overall admission rate, there is an excess of females.

The features in the history of particular value were the difficulties in reconciling the severity of the paralysis with the vagueness of the initially presented incident and the absence of appropriate associated trauma. The findings on physical examination that were helpful have all been reported previously ${ }^{1-9}$ and no new features were found. Of greatest value were preservation of normal tone and reflexes and flexor plantar responses despite apparent weakness. This was accompanied by bizarre posturing and simultaneous contraction of the agonist and antagonist muscles. Within the sensory examination, variable, nonanatomical and inconsistent sensory manifestations was of next value. The most difficult physical finding to interpret was inconsistency between the demonstrated signs and observed function or behaviour, especially in the motor and autonomic fields, since this was dependent on the knowledge and skill of the examining doctor. The non organic patients did not just have one anomalous feature alone; usually at least two were present in each system examined. Owing to the subjective nature of sensory testing, sensory findings were not as marked as those in the motor system. Care has to be taken that repeated examinations, especially by different or inexperienced doctors, does not reinforce disability. ${ }^{9-11}$

The hallmarks of the control group were either preservation of some sensory modality or recovery of sensation preceding motor recovery. The sensory component had the anatomical features consistent with spinal cord injury, that is, dissociation between the different columns and sacral sparing. Difficulties also arise where there is definite evidence of neurological disease but disproportionate disabil- ity. ${ }^{12-17}$ We have therefore kept all such cases out of the series.

We evaluated the role of investigations in assessment, especially as new investigations have appeared since the war series of non organic paraplegia. Plain films are essential. The majority of non organic lesions have normal films and abnormalities if found are of long standing and not associated with trauma. The occasional case where an abnormality which might be relevant is found needs further investigation. Where strong clinical evidence for a non organic lesion existed, the investigation was negative. In contrast, the control series all had an obvious abnormality in either the bones, ligaments or other soft tissues, eg prevertebral soft tissue swelling. Spinal evoked responses in the three cases we have performed have been conclusive insofar as they have been normal, and the parallel EMG studies have shown agonist/ antagonist contractions. CSF examination has been unhelpful but no tests have been performed since assays of oligoclonal bands have become available. Myelography gave no new information in any case where it was undertaken. The indications for the studies performed outside our units was unclear (other than as part of routine assessment). In the two cases in which we undertook myelography, while justifiable because of potentially sinister bony abnormalities and recurrent autonomic disturbance, we would today have resorted to CT scanning and urodynamic stud ies. It must remain a reserve tool for cases where the only support for a non organic diagnosis is a lack of positive abnormal neurological findings or where suspicion is raised because of abnormal investigations or events on follow up in units where other investigations are not available. However, the procedure is not without hazard: patients have developed neurological signs subsequently and adhesive arachnoiditis may occur; this would be disastrous if no organic lesion were present. Indeed, we have seen seven such cases which will be reported separately.

It has been argued that a non organic diagnosis should have positive features in the psychiatric or psychological history or examination and we would support this. All the non organic patients had at least two such features in their histories. The features we found useful, in descending order, were self discharge by walking out, previous functional disturbance, ${ }^{1-379111819}$ a model for paralysis in either their own or their family/friend's histories, and the presence of secondary gain. ${ }^{71013172021}$ The first of these is pathognemonic. Previous functional disturbance occurred in $60 \%$ of cases and in just under $50 \%$ involved the central nervous system. Thirty per cent had a "model" on which to base their illness. Both males and females had evidence of secondary gain but this may not be significant where the gain is 
a civil action, for negligence, because of a similar high incidence in organic paraplegia. In our control series, however, only four civil actions were pending. The association with crime (other than motoring offences) is higher with an overrepresentation among criminals and their victims ( 5 out of 13 male cases). In the controls, there were only two motoring offences, sufficiently minor to be disposed of by post.

The association with active matrimonial proceedings in women is significant; but the overall divorce rate and matrimonial disharmony is no higher between groups and may be no higher than in the community at large. We may have been fortunate in our controls as there were no problems extant (and none arose during treatment) from marital disharmony. The association between non organic paraplegia and "health" occupations in women is previously unreported. Although the NHS is the largest employer in the country, 5 out of 23 is a disproportionate representation. No such association was seen in the control group, even when both sexes were considered. Although there is a $30 \%$ incidence of gynaecological procedures in women ${ }^{22}$ and $25 \%$ of our patients had been psychiatric inpatients at some stage, ${ }^{19}$ these aspects may not be helpful and could be misleading, even though their incidence was higher than in our control group where only two psychiatric past histories were obtained (one of which was long inactive and in the other the psychiatric condition induced the cord injury).

We found any relationship with intelligence, the presence of "belle indifference" or circumscribed memory deficit, and subjective comments by doctors on the quality of the patient's history totally unhelpful. ${ }^{3101123-25}$ Sexual history was unhelpful; many patients in both groups had problems with potency of libido, albeit transient, before injury. Few patients, in either group, reported impotence as a feature. The recovery in incomplete lesions also lagged behind improvement in other autonomic parameters.

The response to treatment was rapid, with complete recovery from symptoms, usually within days, and improvement commenced within hours. The latter can also be seen in organic lesions, some patients produced complete functional recovery in similar times, but overall, the non organic recovered faster. The pattern of recovery (global improvement simultaneously occurring in motor, sensory and autonomic systems) in the non organic group was different from that in the organic group. In the organic group sensory recovery appeared to occur before motor. Further, there was always some residual objective sign of neurological disturbance in organic lesions, and a majority had some persistent autonomic disturbance, although the pattern was always one of improvement (not deterioration) during follow up.
The most successful management of the non organic lesion seemed to be that of a firm diagnosis, a confident prediction of improvement and "sympathy, interest and common sense" advocated by Barham Carter. We have found that showing patients their normal spinal evoked responses will assist in recovery. Any florid theatrical performances should be ignored. Patients should be seen, if possible, as outpatients rather than as inpatients. If admitted (as is usually the case where the referring hospital has no suspicion of a non organic lesion), once the suspicion has been raised, the patient should be nursed in a side ward away from ward routine, which may induce acquired behaviour. They must not be forgotten and a firm directed course of exercises seeking improvement with each session imposed.

Of the series, excepting those with major psychiatric disease, only four went on to develop further functional disturbances through two indulged in attention seeking behaviour. In these patients psychiatric intervention may be needed subsequently. ${ }^{71519}$ In the control group, without preexisting psychiatric disease, psychiatric intervention was never required and in the one pre-existing psychiatric case, these features became better and not worse. It has not been our practice to routinely seek a psychiatric opinion in non organic cases.

Over two thirds of the non organic group have been seen or heard of on follow up, and none have been found to harbour organic neurological disease. We feel, therefore, that a diagnosis of non organic paraplegia can be arrived at using clinical criteria coupled with simple investigations. Positive abnormalities will be found on physical examination in addition to a mere absence of conventional neurological abnormal signs, as will evidence of psychological features. More than one abnormal feature will be found. The diagnosis involves both neurological and psychiatric criteria. ${ }^{46-29}$ Difficulties will occur if attempts are made to use only one or the other. ${ }^{121430-32}$ With such features, the diagnosis can be confidently made ${ }^{33}$ and any residual doubt can be removed by evoked responses $^{34}$ and urodynamic studies and, rarely, CT scanning. Myelography is not required for assessment, today and above all not as a routine to exclude neurological disease. Those with organic lesions will have abnormal neurological signs and abnormal plain radiological findings. Without markers for a non organic lesion and without psychological features, the diagnosis of a functional disorder is incorrect.

\section{References}

1 Carden NL, Schamel DJ. Observations of concersion reactions seen in troops involved in Vietnam conflict. Am J Psychiatry 1966;123:21-31. 
2 Ziegler D. Neurological disease and hysteria: the differential diagnosis. International $J$ Neuropsychiatry 1967;3:388-96.

3 Green MA. Neurological manifestations of conversion hysteria. Trans Am Neurol Assoc 1955;86:196-8.

4 Ziegler FJ. Hysterical conversion reactions. Postgrad Med 1970;47:174-8.

5 Weiser HI. Motor sensory dysfunction of the upper limb due to conversion syndrome. Arch Phys Med Rehab 1976;57:17-9.

6 Rogoff JB, Chrystal M. Hysterical paralysis diagnosed as poliomyelitis. Physical Therapy Reviews 1952:331-7.

7 Friedman SB. Conversion symptoms in adolescents. Pediatr Clin North Am 1973;20:873-82.

8 Worden RE, Johnson EW, Burk RD. Diagnosis of hysterical paralysis. Arch Phys Med Rehab 1961;42:122-3.

9. Lightburn JL, Cattell RB, Stephenson WF. Differential diagnosis of conversion reactions in a general hospital. Postgrad Med 1958;23:140-7.

10 O'Connor PJ. Hysterical reactions. Practitioner 1973;210:58-64.

11 Stevens H. Conversion hysteria: a neurologic emergency. Mayo Clin Proc 1968;43:54-64.

12 Althaus J. On Epilepsy, Hysteria and Ataxy. London: John Churchill and Sons, 1866.

13 Slater E. Shorvon Memorial Lecture. Diagnosis of "hysteria". Br Med J 1965;1395-9.

14 Slater E. The 35th Maudsley Lecture. "Hysteria 311". J Men Sci 1961;107:359.

15 Skey FC. Hysteria. London: Longmans \& Co, 1867.

16 Babinski J. Definition de l'hysterie. Soc Neurol Paris. 7 Novembre 1901.

17 Gowers WR. A Manual of Diseases of the Nervous System. I \& II. London: J \& A Churchill, 1886-88.

18 Stevens $\mathrm{H}$. Conversion hysteria: a therapeutic opportunity for the neurologist. Trans Am Neurol Assoc 1971;96:313-4.
19 Kirschner LA, Kaplan N. Conversion as a manifestation of crisis in the life situation. Compr Psychiatry 1970;11:260-88.

20 Meyer-Gross W, Slater E, Roth M. Clinical Psychiatry. 3 ed London: Cassell \& Co, 1969.

21 Rabkin R. Conversion hysteria as social maladaption. Psychiatry 1964;27:349-63.

22 Cohen ME, Robins E, Purtell JJ, Altman MW. Excessive surgery in hysteria: a study of surgical procedures in 50 women with hysteria and 190 controls. JAMA 1953;151:977-86.

23 Chodoff $\mathbf{P}$, Lyons $\mathbf{H}$. Hysteria, the hysterical personality and hysterical conversion. Am $J$ Psychiatry 1958;114:734-40.

24 Ljungberg L. Hysteria. Acta Psych Scand 1957;Suppl 112.

25 Aring CD. Observations on multiple sclerosis and conversion hysteria. Brain 1965;88:663-74.

26 Merskey H. The Analysis of Hysteria. London: Bailiere Tindall, 1979.

27 Head H, Riddoch G. In: Head H, ed. Studies in Neurology. London: Hodder, 1920:466-530. Originally published in: Brain 1917;XL:188-263.

$28 \mathrm{Head} \mathrm{H}$. An address on the diagnosis of hysteria. $\mathrm{Br}$ Med $J$ 1922;i:827-9.

29 Arietti S. Adult Clinical Psychiatry. American Handbook of Psychiatry. 2nd ed Vol 3. New York: Basic Books, 1974:166-8.

30 Sydenham T. In: Veith I, ed. Hysteria: a History of the Disease. Chicago: Chicago University Press, 1965.

31 Wilson SAK. Neurology 1940;1:156.

32 Sebastianpillai FJY, Wijesinghe CP. Hysterical paraly sis. Ceylon Medical Journal 1972;7:75-9.

33 Barham Carter A. The prognosis of certain hysterical symptoms. Br Med J 1949;1:1253-4.

34 Levy R, Mushin J. Somatosensory evoked response in patients with hysterical anaesthesia. $J$ Psychosom Res 1973;17:81-4. 